\title{
Eigenvalue bounds of the shift-splitting preconditioned singular nonsymmetric saddle-point matrices
}

\author{
Quan Shi ${ }^{1}$, Qin-Qin Shen ${ }^{1,2^{*}}$ and Lin-Quan Yao ${ }^{2}$
}

\section{"Correspondence:}

shenqq@ntu.edu.cn

${ }^{1}$ School of Transportation, Nantong

University, Nantong, 226019, P.R.

China

${ }^{2}$ School of Urban Rail

Transportation, Soochow University,

Suzhou, 215006, P.R. China

\begin{abstract}
For singular nonsymmetric saddle-point problems, a shift-splitting preconditioner was studied in (Appl. Math. Comput. 269:947-955, 2015). To further show the efficiency of the shift-splitting preconditioner, we provide eigenvalue bounds for the nonzero eigenvalues of the shift-splitting preconditioned singular nonsymmetric saddle-point matrices. For real parts of the eigenvalues, the bound is provided by valid inequalities. For eigenvalues having nonzero imaginary parts, the bound is a combination of two inequalities proving their clustering in a confined region of the complex plane. Finally, two numerical examples are presented to verify the theoretical results.
\end{abstract}

MSC: $65 F 10$

Keywords: singular nonsymmetric saddle-point matrix; shift-splitting preconditioner; eigenvalue

\section{Introduction}

Consider the large and sparse nonsymmetric saddle point problems

$$
\mathcal{A} u \equiv\left[\begin{array}{cc}
A & B^{T} \\
-B & 0
\end{array}\right]\left[\begin{array}{l}
x \\
y
\end{array}\right]=\left[\begin{array}{l}
f \\
g
\end{array}\right] \equiv b,
$$

where $A=A^{T} \in \mathbb{R}^{n \times n}$ is positive definite, $B \in \mathbb{R}^{m \times n}$ is a rectangular matrix with $m \leq n$, and $u=\left[x^{T}, y^{T}\right]^{T}$ and $b=\left[f^{T}, g^{T}\right]^{T}$ with $x, f \in \mathbb{R}^{n}$ and $y, g \in \mathbb{R}^{m}$ are the unknown and given right-hand side vectors, respectively. The system of linear equations (1.1) arises from many scientific computing and engineering applications, for example, the mixed finite element discretization of the Stokes equation [2], the Lagrange-type methods for constrained optimization problems [3], the meshfree discretization of elastic mechanics equation [4], and so on. For more background information on the applications of saddle-point problems, see $[5,6]$ and references therein. According to the algebraic properties of the saddle-point matrix $\mathcal{A}[5,7]$, we know that the linear system (1.1) is nonsingular if the matrix $B$ is of full row $\operatorname{rank}($ i.e., $\operatorname{rank}(B)=m$ ) and is singular if the matrix $B$ is rank deficient (i.e., $\operatorname{rank}(B)<m$ ). In this paper, we consider the singular case and always assume that $\operatorname{rank}(B)=r<m$ and that the singular nonsymmetric saddle-point problem (1.1) is consistent, that is, $b \in \operatorname{range}(\mathcal{A})$.

(c) 2016 Shi et al. This article is distributed under the terms of the Creative Commons Attribution 4.0 International License (http://creativecommons.org/licenses/by/4.0/), which permits unrestricted use, distribution, and reproduction in any medium, provided you give appropriate credit to the original author(s) and the source, provide a link to the Creative Commons license, and indicate if changes were made. 
Due to its property of large sparsity, the nonsymmetric saddle-point problem (1.1) is suitable for solving by iterative methods. To get fast convergence rate of the iterative methods, preconditioning techniques are often used [8]. The term preconditioning is simply a means of transforming the original linear system into one that has the same solution, but which is likely to be easier to solve with an iterative solver. A lot of efficient preconditioning techniques have been studied in the past few decades; see [9] for a good survey of efficient preconditioners for saddle-point problems. In this paper, we focus on the following shift-splitting (SS) preconditioner [1, 10]:

$$
\hat{\mathcal{P}}_{S S}=\frac{1}{2}\left[\begin{array}{cc}
\alpha I_{n}+A & B^{T} \\
-B & \beta I_{m}
\end{array}\right] \text {, }
$$

where $\alpha$ and $\beta$ are two positive parameters, and $I_{n}$ and $I_{m}$ are the $n \times n$ and $m \times m$ identity matrices, respectively. In fact, the shift-splitting preconditioner can be induced by the following shift-splitting iteration method:

$$
\frac{1}{2}\left[\begin{array}{cc}
\alpha I_{n}+A & B^{T} \\
-B & \beta I_{m}
\end{array}\right]\left[\begin{array}{l}
x_{k+1} \\
y_{k+1}
\end{array}\right]=\frac{1}{2}\left[\begin{array}{cc}
\alpha I_{n}-A & -B^{T} \\
B & \beta I_{m}
\end{array}\right]\left[\begin{array}{l}
x_{k} \\
y_{k}
\end{array}\right]+\left[\begin{array}{l}
f \\
g
\end{array}\right] .
$$

Theoretical results in [10] and [1] show that the shift-splitting iteration method (1.3) is convergent and semiconvergent unconditionally for solving nonsingular and singular nonsymmetric saddle-point problems, respectively. In the particular case $\alpha=0$, the eigenvalue distribution of the shift-splitting preconditioned nonsingular saddle-point matrix $\hat{\mathcal{P}}_{S S}^{-1} \mathcal{A}$ was studied in [10]. Note that, as a preconditioner, the prefactor $\frac{1}{2}$ in the matrix $\hat{\mathcal{P}}_{S S}$ has no effect on the preconditioned system. Thus, generally, we can use

$$
\mathcal{P}_{S S}=2 \hat{\mathcal{P}}_{S S}=\left[\begin{array}{cc}
\alpha I_{n}+A & B^{T} \\
-B & \beta I_{m}
\end{array}\right]
$$

instead of the original shift-splitting preconditioner $\hat{\mathcal{P}}_{S S}$ (1.2). Although $\mathcal{P}_{S S}$ is not induced by the iteration scheme (1.3), the computational complexities of the preconditioners $\mathcal{P}_{S S}$ and $\hat{\mathcal{P}}_{S S}$ are almost the same. Numerical results in $[1,10]$ show that the shift-splitting preconditioner (1.2) or (1.4) is very efficient. For an overview of the shift-splitting preconditioner (1.2) and the corresponding shift-splitting iteration method (1.3), see recent papers $[11,12]$ and references [13-15].

Since the spectral distribution of the preconditioned matrix is closely related to the convergence rate of Krylov subspace iteration methods [8], we hope that the resulting preconditioned saddle-point matrices have desired eigenvalue distributions, that is, tightly clustered spectra or positive real spectra, and so on; see, for example, [7, 16-19]. To further show the efficiency of the shift-splitting preconditioner (1.2) and (1.3), in this paper, we provide eigenvalue bounds for the nonzero eigenvalues of the shift-splitting preconditioned singular nonsymmetric saddle-point matrices $\mathcal{P}_{S S}^{-1} \mathcal{A}$ that depend only on the extremal eigenvalues of $A$ and nonzero extremal singular values of $B$. We show that all eigenvalues having nonzero imaginary parts are located in an intersection of two circles and all nonzero real eigenvalues are located in a positive interval. Although the analysis is done for the singular case, the theoretical results of the nonsingular case can be obtained as 
a particular case. These theoretical results are presented in Section 2. In Section 3, we present two numerical examples to verify our theoretical results. Finally, in Section 4, we end this paper with a few concluding remarks.

\section{Eigenvalue bounds of the shift-splitting preconditioned matrix $\mathcal{P}_{s s}^{-1} \mathcal{A}$}

In this section, we study eigenvalue bounds of the shift-splitting preconditioned saddlepoint matrix $\mathcal{P}_{S S}^{-1} \mathcal{A}$. For convenience, we first present some notation. For square matrices $M$ and $N, M(\preceq) \prec N$ means that $N-M$ is symmetric positive (semi)definite; (.)* denotes the conjugate transpose of either a vector or a matrix; $\bar{\theta}$ stands for the conjugate of a complex number $\theta ; \operatorname{Re}(\theta)$ and $\operatorname{Im}(\theta)$ stand for the real and imaginary parts of a complex number $\theta ; \rho(M)$ and null $(M)$ denote the spectral radius and the null space of the matrix $M$, respectively. Let the eigenvalues of the symmetric positive definite matrix $A$ be

$$
\eta_{1} \geq \eta_{2} \geq \cdots \geq \eta_{n}>0
$$

and the singular values of the matrix $B$ be

$$
\sigma_{1} \geq \sigma_{2} \geq \cdots \geq \sigma_{r}>\sigma_{r+1}=\cdots=\sigma_{m}=0 .
$$

We first present the eigenvalue distribution of the shift-splitting preconditioned matrix $\mathcal{P}_{S S}^{-1} \mathcal{A}$ and give a bound for its nonzero eigenvalues. To this end, we first give a useful lemma.

Lemma 2.1 ([11]) Assume that $A \in \mathbb{R}^{n \times n}$ is positive definite and $B \in \mathbb{R}^{m \times n}(m \leq n)$ has full row rank. Let the iteration matrix $\Gamma$ of the shift-splitting iteration (1.3) be

$$
\Gamma=\left[\begin{array}{cc}
\alpha I_{n}+A & B^{T} \\
-B & \beta I_{m}
\end{array}\right]^{-1}\left[\begin{array}{cc}
\alpha I_{n}-A & -B^{T} \\
B & \beta I_{m}
\end{array}\right] .
$$

Then

$$
\rho(\Gamma)<1, \quad \forall \alpha, \beta>0 .
$$

Theorem 2.1 Assume that $A \in \mathbb{R}^{n \times n}$ is symmetric positive definite and $B \in \mathbb{R}^{m \times n}(m \leq n)$ is rank deficient with $\operatorname{rank}(B)=r<m$. Let the shift-splitting preconditioner $\mathcal{P}_{S S}$ be defined as in (1.4), and $\alpha, \beta>0$. Then the shift-splitting preconditioned matrix $\mathcal{P}_{S S}^{-1} \mathcal{A}$ has $m-r$ zero eigenvalue values and $n+r$ nonzero eigenvalues. Let $\lambda$ be a nonzero eigenvalue of the shift-splitting preconditioned matrix $\mathcal{P}_{S S}^{-1} \mathcal{A}$. Then it satisfies

$$
\left|\lambda-\frac{1}{2}\right|<\frac{1}{2}
$$

that is, all nonzero eigenvalues are located inside the circle centered at $\left(\frac{1}{2}, 0\right)$ with radius $\frac{1}{2}$.

Proof From (2.2) we assume that the singular value decomposition of the matrix $B$ is

$$
B=U\left[\begin{array}{c}
\tilde{B} \\
0
\end{array}\right] V^{T},
$$


where $U \in \mathbb{R}^{m \times m}$ and $V \in \mathbb{R}^{n \times n}$ are orthogonal matrices, and $\tilde{B}=\left[\Sigma_{r} 0\right] \in \mathbb{R}^{r \times n}$ is of full row rank with $\Sigma_{r}=\operatorname{diag}\left(\sigma_{1}, \sigma_{2}, \ldots, \sigma_{r}\right) \in \mathbb{R}^{r \times r}$.

By the definition of the shift-splitting preconditioner we have

$$
\begin{aligned}
& \mathcal{P}_{S S}^{-1} \mathcal{A}=\frac{1}{2} \hat{\mathcal{P}}_{S S}^{-1} \mathcal{A} \\
& =\frac{1}{2}\left(I_{n+m}-\Gamma\right) \\
& =\frac{1}{2} I_{n+m}-\frac{1}{2}\left[\begin{array}{cc}
\alpha I_{n}+A & B^{T} \\
-B & \beta I_{m}
\end{array}\right]^{-1}\left[\begin{array}{cc}
\alpha I_{n}-A & -B^{T} \\
B & \beta I_{m}
\end{array}\right] \\
& =\frac{1}{2} I_{n+m}-\frac{1}{2}\left[\begin{array}{cc}
V & 0 \\
0 & U
\end{array}\right]\left[\begin{array}{ccc}
\alpha I_{n}+V^{T} A V & \tilde{B}^{T} & 0 \\
-\tilde{B} & \beta I_{r} & 0 \\
0 & 0 & \beta I_{m-r}
\end{array}\right]^{-1} \\
& \times\left[\begin{array}{ccc}
\alpha I_{n}-V^{T} A V & -\tilde{B}^{T} & 0 \\
\tilde{B} & \beta I_{r} & 0 \\
0 & 0 & \beta I_{m-r}
\end{array}\right]\left[\begin{array}{cc}
V^{T} & 0 \\
0 & U^{T}
\end{array}\right],
\end{aligned}
$$

which is similar to

$$
\left[\begin{array}{cc}
\frac{1}{2} I_{n+r}-\frac{1}{2} \tilde{\Gamma} & 0 \\
0 & 0
\end{array}\right],
$$

where

$$
\tilde{\Gamma}=\left[\begin{array}{cc}
\alpha I_{n}+V^{T} A V & \tilde{B}^{T} \\
-\tilde{B} & \beta I_{r}
\end{array}\right]^{-1}\left[\begin{array}{cc}
\alpha I_{n}-V^{T} A V & -\tilde{B}^{T} \\
\tilde{B} & \beta I_{r}
\end{array}\right] .
$$

Since $V$ is an orthogonal matrix and $A$ is symmetric positive definite, $V^{T} A V$ is symmetric positive definite, too. From (2.4) we know that $\tilde{B}$ is of full row rank. Thus, by Lemma 2.1 we have $\rho(\tilde{\Gamma})<1$. Therefore, from (2.5) we obtain that the shift-splitting preconditioned matrix $\mathcal{P}_{S S}^{-1} \mathcal{A}$ has $m-r$ zero eigenvalue values and $n+r$ nonzero eigenvalues.

Now, we study a bound for the nonzero eigenvalues of the preconditioned matrix $\mathcal{P}_{S S}^{-1} \mathcal{A}$. Let $\lambda$ and $\theta$ be nonzero eigenvalues of the shift-splitting preconditioned matrix $\mathcal{P}_{S S}^{-1} \mathcal{A}$ and $\tilde{\Gamma}$, respectively. Then we have

$$
\lambda=\frac{1}{2}-\frac{1}{2} \theta \quad \text { and } \quad|\theta|<1 .
$$

By direct computation we obtain

$$
\left|\lambda-\frac{1}{2}\right|<\frac{1}{2}
$$

that is, all nonzero eigenvalues of the shift-splitting preconditioned matrix $\mathcal{P}_{S S}^{-1} \mathcal{A}$ are located inside the circle centered at $\left(\frac{1}{2}, 0\right)$ with radius $\frac{1}{2}$.

In the following, we further derive bounds for the nonreal and real eigenvalues of the shift-splitting preconditioned matrix $\mathcal{P}_{S S}^{-1} \mathcal{A}$. Based on the singular value decomposition 
(2.4) and the techniques used in Theorem 2.1, we can see that the shift-splitting preconditioned matrix $\mathcal{P}_{S S}^{-1} \mathcal{A}$ can be rewritten as

$$
\begin{aligned}
& \mathcal{P}_{S S}^{-1} \mathcal{A}=\left[\begin{array}{cc}
\alpha I_{n}+A & B^{T} \\
-B & \beta I_{m}
\end{array}\right]^{-1}\left[\begin{array}{cc}
A & B^{T} \\
-B & 0
\end{array}\right]
\end{aligned}
$$

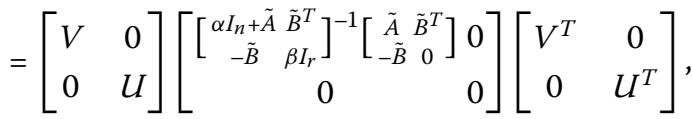

where $\tilde{A}=V^{T} A V$, and $\tilde{B}$ is defined as in (2.4). Let

$$
\tilde{\mathcal{P}}_{S S}^{-1} \tilde{\mathcal{A}}=\left[\begin{array}{cc}
\alpha I_{n}+\tilde{A} & \tilde{B}^{T} \\
-\tilde{B} & \beta I_{r}
\end{array}\right]^{-1}\left[\begin{array}{cc}
\tilde{A} & \tilde{B}^{T} \\
-\tilde{B} & 0
\end{array}\right] .
$$

Equality (2.6) indicates that the nonzero eigenvalues of the shift-splitting preconditioned matrix $\mathcal{P}_{S S}^{-1} \mathcal{A}$ are the eigenvalues of $\tilde{\mathcal{P}}_{S S}^{-1} \tilde{\mathcal{A}}$.

To further derive a bound for the nonreal eigenvalues of the shift-splitting preconditioned matrix $\mathcal{P}_{S S}^{-1} \mathcal{A}$, we use the following lemma. Note that the conclusion of the lemma is a particular case of [20], Theorem 2 .

Lemma 2.2 ([20]) Assume that $A \in \mathbb{R}^{n \times n}$ is positive definite and $B \in \mathbb{R}^{m \times n}(m \leq n)$ has full row rank. Let

$$
\mathcal{A}_{+}=\left[\begin{array}{cc}
A & B^{T} \\
B & 0
\end{array}\right],
$$

and let

$$
\mathcal{P}_{B T}=\left[\begin{array}{cc}
\hat{A} & B^{T} \\
0 & -\hat{C}
\end{array}\right]
$$

be a block triangular preconditioner to $\mathcal{A}_{+}$, where $\hat{A} \in \mathbb{R}^{n \times n}$ and $\hat{C} \in \mathbb{R}^{m \times m}$ are symmetric positive definite matrices. Let $\theta$ be an eigenvalue of the block triangular preconditioned matrix $\mathcal{P}_{B T}^{-1} \mathcal{A}_{+}$, and $\tau_{n}$ be the smallest eigenvalue of the symmetric positive definite matrix $\hat{A}^{-\frac{1}{2}} A \hat{A}^{-\frac{1}{2}}$. If $1-\tau_{n} \geq 0$, then

$$
|\theta-1| \leq \sqrt{1-\tau_{n}} .
$$

If $1-\tau_{n}<0$, then all eigenvalues of the block triangular preconditioned matrix $\mathcal{P}_{B T}^{-1} \mathcal{A}_{+}$are real.

Theorem 2.2 Assume that $A \in \mathbb{R}^{n \times n}$ is symmetric positive definite and $B \in \mathbb{R}^{m \times n}(m \leq n)$ is rank deficient with $\operatorname{rank}(B)=r<m$. Let the shift-splitting preconditioner $\mathcal{P}_{S S}$ be defined as in (1.4), and $\alpha, \beta>0$. Let $\lambda$ be a nonzero eigenvalue having nonzero imaginary part of the shift-splitting preconditioned matrix $\mathcal{P}_{\text {SS }}^{-1} \mathcal{A}$. Then it satisfies

$$
|\lambda-1| \leq \sqrt{\frac{\alpha}{\alpha+\eta_{n}}},
$$

where $\eta_{n}$ is the smallest eigenvalue of the matrix $A$. 
Proof By the preceding discussions, only the eigenvalues of the matrix $\tilde{\mathcal{P}}_{S S}^{-1} \tilde{\mathcal{A}}(2.7)$ need to be studied. Consider the additional auxiliary block diagonal symmetric positive definite matrix

$$
\tilde{\mathcal{F}}=\left[\begin{array}{cc}
\alpha I_{n}+\tilde{A} & 0^{T} \\
0 & \beta I_{r}
\end{array}\right]
$$

Then the matrix $\tilde{\mathcal{P}}_{S S}^{-1} \tilde{\mathcal{A}}$ is similar to

$$
\begin{aligned}
\left(\tilde{\mathcal{F}}^{-\frac{1}{2}} \tilde{\mathcal{P}}_{S S} \tilde{\mathcal{F}}^{-\frac{1}{2}}\right)^{-1} \tilde{\mathcal{F}}^{-\frac{1}{2}} \tilde{\mathcal{A}} \tilde{\mathcal{F}}^{-\frac{1}{2}} & =\left[\begin{array}{cc}
I_{n} & \bar{B}^{T} \\
-\bar{B} & I_{r}
\end{array}\right]^{-1}\left[\begin{array}{cc}
\bar{A} & \bar{B}^{T} \\
-\bar{B} & 0
\end{array}\right] \\
& =\left[\begin{array}{cc}
I_{n} & \bar{B}^{T} \\
\bar{B} & -I_{r}
\end{array}\right]^{-1}\left[\begin{array}{cc}
\bar{A} & \bar{B}^{T} \\
\bar{B} & 0
\end{array}\right],
\end{aligned}
$$

where $\bar{A}=\left(\alpha I_{n}+\tilde{A}\right)^{-\frac{1}{2}} \tilde{A}\left(\alpha I_{n}+\tilde{A}\right)^{-\frac{1}{2}}$ and $\bar{B}=\frac{1}{\sqrt{\beta}} \tilde{B}\left(\alpha I_{n}+\tilde{A}\right)^{-\frac{1}{2}}$. Since

$$
\left[\begin{array}{cc}
I_{n} & \bar{B}^{T} \\
\bar{B} & -I_{r}
\end{array}\right]=\left[\begin{array}{cc}
I_{n}+\bar{B}^{T} \bar{B} & \bar{B}^{T} \\
0 & -I_{r}
\end{array}\right]\left[\begin{array}{cc}
I_{n} & 0 \\
-\bar{B} & I_{r}
\end{array}\right],
$$

we can rewrite the right-hand side of (2.8) as

$$
\left[\begin{array}{cc}
I_{n} & 0 \\
-\bar{B} & I_{r}
\end{array}\right]^{-1}\left[\begin{array}{cc}
I_{n}+\bar{B}^{T} \bar{B} & \bar{B}^{T} \\
0 & -I_{r}
\end{array}\right]^{-1}\left[\begin{array}{cc}
\bar{A} & \bar{B}^{T} \\
\bar{B} & 0
\end{array}\right]
$$

which is similar to

$$
\begin{aligned}
& {\left[\begin{array}{cc}
I_{n}+\bar{B}^{T} \bar{B} & \bar{B}^{T} \\
0 & -I_{r}
\end{array}\right]^{-1}\left[\begin{array}{cc}
\bar{A} & \bar{B}^{T} \\
\bar{B} & 0
\end{array}\right]\left[\begin{array}{cc}
I_{n} & 0 \\
-\bar{B} & I_{r}
\end{array}\right]^{-1}} \\
& \quad=\left[\begin{array}{cc}
I_{n}+\bar{B}^{T} \bar{B} & \bar{B}^{T} \\
0 & -I_{r}
\end{array}\right]^{-1}\left[\begin{array}{cc}
\bar{A}+\bar{B}^{T} \bar{B} & \bar{B}^{T} \\
\bar{B} & 0
\end{array}\right] \\
& \triangleq \overline{\mathcal{P}}_{S S}^{-1} \overline{\mathcal{A}} .
\end{aligned}
$$

Therefore, the preconditioned matrix $\tilde{\mathcal{P}}_{S S}^{-1} \tilde{\mathcal{A}}$ is similar to the matrix $\overline{\mathcal{P}}_{S S}^{-1} \overline{\mathcal{A}}$, and we only need to provide the eigenvalue bounds for $\overline{\mathcal{P}}_{S S}^{-1} \overline{\mathcal{A}}$.

By Lemma 2.2 we need to study the smallest eigenvalue of the matrix $\left(I_{n}+\bar{B}^{T} \bar{B}\right)^{-\frac{1}{2}}(\bar{A}+$ $\left.\bar{B}^{T} \bar{B}\right)\left(I_{n}+\bar{B}^{T} \bar{B}\right)^{-\frac{1}{2}}$, which is similar to $\left(\alpha I_{n}+\tilde{A}+\frac{1}{\beta} \tilde{B}^{T} \tilde{B}\right)^{-1}\left(\tilde{A}+\frac{1}{\beta} \tilde{B}^{T} \tilde{B}\right)$. Let $\tilde{\tau}$ be an eigenvalue of the matrix $\left(\alpha I_{n}+\tilde{A}+\frac{1}{\beta} \tilde{B}^{T} \tilde{B}\right)^{-1}\left(\tilde{A}+\frac{1}{\beta} \tilde{B}^{T} \tilde{B}\right)$, and $\tilde{v}$ be the corresponding eigenvector. Then we have

$$
\left(\tilde{A}+\frac{1}{\beta} \tilde{B}^{T} \tilde{B}\right) \tilde{v}=\tilde{\tau}\left(\alpha I_{n}+\tilde{A}+\frac{1}{\beta} \tilde{B}^{T} \tilde{B}\right) \tilde{v}
$$

from which we obtain

$$
0<\frac{\eta_{n}}{\alpha+\eta_{n}} \leq \frac{\tilde{v}^{*} \tilde{A} \tilde{v}}{\alpha \tilde{v}^{*} \tilde{v}+\tilde{v}^{*} \tilde{A} \tilde{v}} \leq \tilde{\tau}=\frac{\tilde{v}^{*} \tilde{A} \tilde{v}+\frac{1}{\beta} \tilde{v}^{*} \tilde{B}^{T} \tilde{B} \tilde{v}}{\alpha \tilde{v}^{*} \tilde{v}+\tilde{v}^{*} \tilde{A} \tilde{v}+\frac{1}{\beta} \tilde{v}^{*} \tilde{B}^{T} \tilde{B} \tilde{v}} \leq \frac{\beta \eta_{1}+\sigma_{1}^{2}}{\alpha \beta+\beta \eta_{1}+\sigma_{1}^{2}}<1
$$


Let $\lambda$ be a nonzero eigenvalue of the shift-splitting preconditioned matrix $\mathcal{P}_{S S}^{-1} \mathcal{A}$. Then by Lemma 2.2 we have

$$
|\lambda-1| \leq \sqrt{1-\frac{\eta_{n}}{\alpha+\eta_{n}}}=\sqrt{\frac{\alpha}{\alpha+\eta_{n}}}
$$

which shows that all eigenvalues having nonzero imaginary parts of the preconditioned matrix $\mathcal{P}_{S S}^{-1} \mathcal{A}$ are located in the circle centered at $(1,0)$ with radius $\sqrt{\frac{\alpha}{\alpha+\eta_{n}}}$ strictly less than one.

Theorem 2.2 shows that the bound for the eigenvalues having nonzero imaginary parts of the shift-splitting preconditioned matrix $\mathcal{P}_{S S}^{-1} \mathcal{A}$ only depends on the parameter $\alpha$ and the smallest eigenvalue of the matrix $A$. Combining Theorem 2.1 with Theorem 2.2, we can obtain some refined bounds for the nonreal eigenvalues of the shift-splitting preconditioned matrix $\mathcal{P}_{S S}^{-1} \mathcal{A}$ in the following theorem.

Theorem 2.3 Assume that $A \in \mathbb{R}^{n \times n}$ is symmetric positive definite and $B \in \mathbb{R}^{m \times n}(m \leq n)$ is rank deficient with $\operatorname{rank}(B)=r<m$. Let the shift-splitting preconditioner $\mathcal{P}_{S S}$ be defined as in (1.4), and $\alpha, \beta>0$. Then all eigenvalues $\lambda$ having nonzero imaginary parts of the shiftsplitting preconditioned matrix $\mathcal{P}_{S S}^{-1} \mathcal{A}$ are located in the domain

$$
\left\{\lambda|| \lambda-\frac{1}{2}\left|<\frac{1}{2} \cap\right| \lambda-1 \mid \leq \sqrt{\frac{\alpha}{\alpha+\eta_{n}}}, \lambda \in \mathbb{C}\right\}
$$

where $\eta_{n}$ is the smallest eigenvalue of the matrix $A$.

Theorem 2.4 Assume that $A \in \mathbb{R}^{n \times n}$ is symmetric positive definite and $B \in \mathbb{R}^{m \times n}(m \leq n)$ is rank deficient with $\operatorname{rank}(B)=r<m$. Let the shift-splitting preconditioner $\mathcal{P}_{S S}$ be defined as in (1.4), and $\alpha, \beta>0$. The nonzero eigenvalues $\lambda$ of the shift-splitting preconditioned matrix $\mathcal{P}_{\text {SS }}^{-1} \mathcal{A}$ satisfy

$$
\min \left\{\frac{\eta_{n}}{\alpha+\eta_{n}}, \frac{\sigma_{r}^{2}}{\alpha \beta+\beta \eta_{1}+\sigma_{r}^{2}}\right\} \leq \operatorname{Re}(\lambda) \leq \frac{\beta \eta_{1}+\sigma_{1}^{2}}{\alpha \beta+\beta \eta_{1}+\sigma_{1}^{2}},
$$

where $\eta_{1}$ and $\eta_{n}$ are the largest and the smallest eigenvalues of the matrix $A$, and $\sigma_{1}$ and $\sigma_{r}$ are the largest and smallest nonzero singular values of the matrix $B$, respectively.

Proof According to the proof of Theorem 2.2, we only need to provide bounds for the real eigenvalues of the matrix $\overline{\mathcal{P}}_{S S}^{-1} \overline{\mathcal{A}}$, which is similar to $\overline{\mathcal{A}} \overline{\mathcal{P}}_{S S}^{-1}$. Consider additionally the block diagonal symmetric positive definite matrix

$$
\check{\mathcal{F}}=\left[\begin{array}{cc}
I_{n}+\bar{B}^{T} \bar{B} & 0 \\
0 & I_{r}
\end{array}\right] \text {. }
$$

Then the matrix $\overline{\mathcal{A}} \overline{\mathcal{P}}_{S S}^{-1}$ is similar to

$$
\check{\mathcal{F}}^{-\frac{1}{2}} \overline{\mathcal{A}} \check{\mathcal{F}}^{-\frac{1}{2}}\left(\check{\mathcal{F}}^{-\frac{1}{2}} \overline{\mathcal{P}}_{S S} \check{\mathcal{F}}^{-\frac{1}{2}}\right)^{-1}=\left[\begin{array}{cc}
\check{A} & \check{B}^{T} \\
\check{B} & 0
\end{array}\right]\left[\begin{array}{cc}
I_{n} & \check{B}^{T} \\
0 & -I_{r}
\end{array}\right]^{-1}=\left[\begin{array}{cc}
\check{A} & \left(\check{A}-I_{n}\right) \check{B}^{T} \\
\check{B} & \check{B}^{T}
\end{array}\right],
$$

where $\check{A}=\left(I_{n}+\bar{B}^{T} \bar{B}\right)^{-\frac{1}{2}}\left(\bar{A}+\bar{B}^{T} \bar{B}\right)\left(I_{n}+\bar{B}^{T} \bar{B}\right)^{-\frac{1}{2}}$ and $\check{B}=\bar{B}\left(I_{n}+\bar{B}^{T} \bar{B}\right)^{-\frac{1}{2}}$. 
Let $\check{A}=X \Lambda X^{T}$ be the eigenvalue decomposition of $\check{A}$ with $X$ being orthogonal and $\Lambda$ being diagonal. By the proof of Theorem 2.2 we know that $0 \prec \Lambda<I_{n}$. Thus, the right-hand side matrix of (2.10) is orthogonal similar to

$$
\begin{aligned}
& {\left[\begin{array}{cc}
X & 0 \\
0 & I_{r}
\end{array}\right]^{T}\left[\begin{array}{cc}
\check{A} & \left(\check{A}-I_{n} \check{B}^{T}\right. \\
\check{B} & \check{B} \check{B}^{T}
\end{array}\right]\left[\begin{array}{cc}
X & 0 \\
0 & I_{r}
\end{array}\right]} \\
& =\left[\begin{array}{cc}
\Lambda & \left(\Lambda-I_{n}\right) X^{T} \check{B}^{T} \\
\check{B} X & \check{B} \check{B}^{T}
\end{array}\right] \\
& =\left[\begin{array}{cc}
\Lambda\left(I_{n}-\Lambda\right) & \left(\Lambda-I_{n}\right) X^{T} \check{B}^{T} \\
\check{B} X\left(I_{n}-\Lambda\right) & \check{B} \check{B}^{T}
\end{array}\right]\left[\begin{array}{cc}
I_{n}-\Lambda & 0 \\
0 & I_{r}
\end{array}\right]^{-1},
\end{aligned}
$$

which is further similar to

$$
\left[\begin{array}{cc}
I_{n}-\Lambda & 0 \\
0 & I_{r}
\end{array}\right]^{-\frac{1}{2}}\left[\begin{array}{cc}
\Lambda\left(I_{n}-\Lambda\right) & \left(\Lambda-I_{n}\right) X^{T} \check{B}^{T} \\
\check{B} X\left(I_{n}-\Lambda\right) & \check{B} \breve{B}^{T}
\end{array}\right]\left[\begin{array}{cc}
I_{n}-\Lambda & 0 \\
0 & I_{r}
\end{array}\right]^{-\frac{1}{2}}=\left[\begin{array}{cc}
\Lambda & -Q^{T} \\
Q & S
\end{array}\right]
$$

where $Q=\check{B} X\left(I_{n}-\Lambda\right)^{\frac{1}{2}}$ and $S=\check{B} \check{B}^{T}$.

Let $\lambda$ be a nonzero eigenvalue of the shift-splitting matrix $\mathcal{P}_{S S}^{-1} \mathcal{A}$. Then by similarity we know that $\lambda$ is also an eigenvalue of the matrix $\left[\begin{array}{cc}\Lambda & -Q^{T} \\ Q & S\end{array}\right]$. From [21], Proposition 2.2, we have

$$
\min \left\{\lambda_{\min }(\Lambda), \lambda_{\min }(S)\right\} \leq \operatorname{Re}(\lambda) \leq \max \left\{\lambda_{\max }(\Lambda), \lambda_{\max }(S)\right\}
$$

Inequalities (2.9) indicate that

$$
\frac{\eta_{n}}{\alpha+\eta_{n}} I_{n} \preceq \Lambda \preceq \frac{\beta \eta_{1}+\sigma_{1}^{2}}{\alpha \beta+\beta \eta_{1}+\sigma_{1}^{2}} I_{n} .
$$

Now, we study the extreme eigenvalues of the symmetric positive definite matrix

$$
S=\check{B} \check{B}^{T}=\bar{B}\left(I_{n}+\bar{B}^{T} \bar{B}\right)^{-1} \bar{B}^{T} .
$$

Let the singular value decomposition of the matrix $\bar{B}$ be

$$
\bar{B}=\bar{U}\left[\begin{array}{ll}
\bar{\Sigma}_{r} & 0
\end{array}\right] \bar{V}^{T},
$$

where $\bar{U} \in \mathbb{R}^{r \times r}$ and $\bar{V} \in \mathbb{R}^{n \times n}$ are orthogonal matrices, and $\bar{\Sigma}_{r}=\operatorname{diag}\left(\bar{\sigma}_{1}, \bar{\sigma}_{2}, \ldots, \bar{\sigma}_{r}\right) \in \mathbb{R}^{r \times r}$ is a diagonal matrix with $\bar{\sigma}_{1} \geq \bar{\sigma}_{2} \geq \cdots \geq \bar{\sigma}_{r}>0$ being the singular values of $\bar{B}$. Then

$$
S=\bar{U}\left[\begin{array}{ll}
\bar{\Sigma}_{r} & 0
\end{array}\right]\left[\begin{array}{cc}
I_{r}+\bar{\Sigma}_{r}^{2} & 0 \\
0 & I_{n-r}
\end{array}\right]^{-1}\left[\begin{array}{c}
\bar{\Sigma}_{r} \\
0
\end{array}\right] \bar{U}^{T}=\bar{U} \operatorname{diag}\left(\frac{\bar{\sigma}_{1}^{2}}{1+\bar{\sigma}_{1}^{2}}, \ldots, \frac{\bar{\sigma}_{r}^{2}}{1+\bar{\sigma}_{r}^{2}}\right) \bar{U}^{T}
$$

and

$$
\frac{\bar{\sigma}_{r}^{2}}{1+\bar{\sigma}_{r}^{2}} I_{r} \preceq S \preceq \frac{\bar{\sigma}_{1}^{2}}{1+\bar{\sigma}_{1}^{2}} I_{r}
$$


where $\bar{\sigma}_{1}^{2}$ and $\bar{\sigma}_{r}^{2}$ are the largest and smallest eigenvalues of the matrix $\bar{B} \bar{B}^{T}=\frac{1}{\beta} \tilde{B}\left(\alpha I_{n}+\right.$ $\tilde{A})^{-1} \tilde{B}^{T}$, respectively. By [8], Theorem 1.22 , we have

$$
\frac{\sigma_{r}^{2}}{\beta\left(\alpha+\eta_{1}\right)} \leq \bar{\sigma}_{r}^{2} \leq \bar{\sigma}_{1}^{2} \leq \frac{\sigma_{1}^{2}}{\beta\left(\alpha+\eta_{n}\right)} .
$$

Then from (2.13) we obtain the inequalities

$$
\frac{\sigma_{r}^{2}}{\alpha \beta+\beta \eta_{1}+\sigma_{r}^{2}} I_{r} \preceq S \preceq \frac{\sigma_{1}^{2}}{\alpha \beta+\beta \eta_{n}+\sigma_{1}^{2}} I_{r} .
$$

From inequalities (2.11), (2.12) and (2.14), the nonzero eigenvalues of the shift-splitting preconditioned matrix $\mathcal{P}_{S S}^{-1} \mathcal{A}$ satisfy

$$
\min \left\{\frac{\eta_{n}}{\alpha+\eta_{n}}, \frac{\sigma_{r}^{2}}{\alpha \beta+\beta \eta_{1}+\sigma_{r}^{2}}\right\} \leq \operatorname{Re}(\lambda) \leq \max \left\{\frac{\beta \eta_{1}+\sigma_{1}^{2}}{\alpha \beta+\beta \eta_{1}+\sigma_{1}^{2}}, \frac{\sigma_{1}^{2}}{\alpha \beta+\beta \eta_{n}+\sigma_{1}^{2}}\right\}
$$

Since

$$
\frac{\beta \eta_{1}+\sigma_{1}^{2}}{\alpha \beta+\beta \eta_{1}+\sigma_{1}^{2}}>\frac{\sigma_{1}^{2}}{\alpha \beta+\beta \eta_{n}+\sigma_{1}^{2}}
$$

equalities (2.15) can be further simplified:

$$
\min \left\{\frac{\eta_{n}}{\alpha+\eta_{n}}, \frac{\sigma_{r}^{2}}{\alpha \beta+\beta \eta_{1}+\sigma_{r}^{2}}\right\} \leq \operatorname{Re}(\lambda) \leq \frac{\beta \eta_{1}+\sigma_{1}^{2}}{\alpha \beta+\beta \eta_{1}+\sigma_{1}^{2}} \text {. }
$$

Thus, we complete the proof.

Remark 2.1 We give some remarks on the obtained theoretical results.

(1) Theorem 2.4 also presents a bound for the real eigenvalues of the shift-splitting preconditioned matrix $\mathcal{P}_{S S}^{-1} \mathcal{A}$. Besides, the bound given in Theorem 2.4 is much tighter than that in Theorem 2.1.

(2) Obviously, if $r=m$, then the nonsymmetric saddle-point matrix $\mathcal{A}$ is nonsingular. Then, the theoretical results presented in Theorems 2.1-2.4 can be extended to the nonsingular case.

\section{Numerical experiments}

In this section, we use two numerical examples to verify the estimated eigenvalue bounds of the shift-splitting preconditioned matrices $\mathcal{P}_{S S}^{-1} \mathcal{A}$ shown in Section 2.

Example 3.1 ([1]) The first test singular nonsymmetric saddle-point problem has the following coefficient submatrices:

$$
\begin{aligned}
A & =\left[\begin{array}{cc}
I \otimes T+T \otimes I & 0 \\
0 & I \otimes T+T \otimes I
\end{array}\right] \in \mathbb{R}^{2 q^{2} \times 2 q^{2}}, \\
B^{T} & =\left[\begin{array}{lll}
\hat{B}^{T} & b_{1} & b_{2}
\end{array}\right] \in \mathbb{R}^{2 q^{2} \times\left(q^{2}+2\right)}
\end{aligned}
$$


Table 1 Bounds of nonzero real eigenvalues of the preconditioned matrix $\mathcal{P}_{\text {SS }}^{-1} \mathcal{A}$ for Example 3.1

\begin{tabular}{lll}
\hline $\boldsymbol{( \alpha}, \boldsymbol{\beta})$ & The estimated bound & The exact bound \\
\hline$(1.9683,6.6748)$ & {$[3.4169 \mathrm{e}-4,0.9993]$} & {$[0.0536,0.9991]$} \\
$(7.8732,6.6572)$ & {$[3.4172 \mathrm{e}-4,0.9970]$} & {$[0.0541,0.9966]$} \\
$(13.7782,6.6397)$ & {$[3.4174 \mathrm{e}-4,0.9948]$} & {$[0.0545,0.9940]$} \\
$(19.6831,6.6223)$ & {$[3.4176 \mathrm{e}-4,0.9926]$} & {$[0.0550,0.9915]$} \\
\hline
\end{tabular}

with

$$
\begin{aligned}
\hat{B}^{T} & =\left[\begin{array}{l}
I \otimes F \\
F \otimes I
\end{array}\right] \in \mathbb{R}^{2 q^{2} \times q^{2}}, \quad b_{1}=\hat{B}^{T}\left[\begin{array}{l}
e \\
0
\end{array}\right], \\
b_{2} & =\hat{B}^{T}\left[\begin{array}{l}
0 \\
e
\end{array}\right], \quad e=[1,1, \ldots, 1] \in \mathbb{R}^{q^{2} / 2}
\end{aligned}
$$

and

$$
T=\frac{1}{h^{2}} \cdot \operatorname{tridiag}(-1,2,-1) \in \mathbb{R}^{q \times q}, \quad F=\frac{1}{h} \cdot \operatorname{tridiag}(-1,1,0) \in \mathbb{R}^{q \times q} .
$$

Here, $\otimes$ denotes the Kronecker product symbol, $v$ is a parameter, and $h=\frac{1}{q+1}$ is the discretization meshsize.

For this example, we have $n=2 p^{2}, m=p^{2}+2$, and the total number of variables is $m+$ $n=3 p^{2}+2$. The matrix $B$ is an augmentation of the full rank matrix $\hat{B}$ with two linearly independent vectors $b_{1}$ and $b_{2}$. Since $b_{1}$ and $b_{2}$ are linear combinations of the columns of the matrix $\hat{B}, B$ is a rank-deficient matrix. Thus, the corresponding nonsymmetric saddlepoint matrix $\mathcal{A}$ is singular. In order to verify the theoretical results, we only choose the case $q=16$ and vary the parameters $\alpha$ and $\beta$. We choose four cases for the parameter $\alpha$, that is, $\alpha=0.1 \eta_{n}, 0.4 \eta_{n}, 0.7 \eta_{n}, \eta_{n}$. The parameter $\beta$ is obtained by the formula $\beta=\|B\|_{2}^{2} / \| \alpha I_{n}+$ $A \|_{2}$, which is often used in the shift-splitting preconditioners $[11,15]$.

The parameters $\alpha$ and $\beta$, the estimated bounds, and the exact bounds of nonzero real eigenvalues of the shift-splitting preconditioned matrices $\mathcal{P}_{S S}^{-1} \mathcal{A}$ are listed in Table 1 . In Figure 1, we plot all eigenvalues of the shift-splitting preconditioned matrix $\mathcal{P}_{S S}^{-1} \mathcal{A}$ for Example 3.1. In particular, the nonreal eigenvalues are plotted by ' $*$ ', and the real eigenvalues are plotted by ' $?$

By actual computation we know that two eigenvalues of the shift-splitting preconditioned matrix $\mathcal{P}_{S S}^{-1} \mathcal{A}$ are zero, which confirms Theorem 2.1. From Table 1 and Figure 1 we can see that the estimated bounds sharply contain the exact bounds of real eigenvalues of $\mathcal{P}_{G S S}^{-1} \mathcal{A}$ and all eigenvalues having nonzero imaginary parts are located in an intersection of two circles. These results are in good agreement with our theoretical results in Theorem 2.3 and Theorem 2.4.

Example $3.2([2,22])$ The second test singular nonsymmetric saddle-point problem arises from the following Stokes equation with suitable boundary conditions:

$$
\left\{\begin{array}{l}
-v \Delta u+\nabla p=f, \quad \text { in } \Omega, \\
\nabla \cdot u=0,
\end{array}\right.
$$




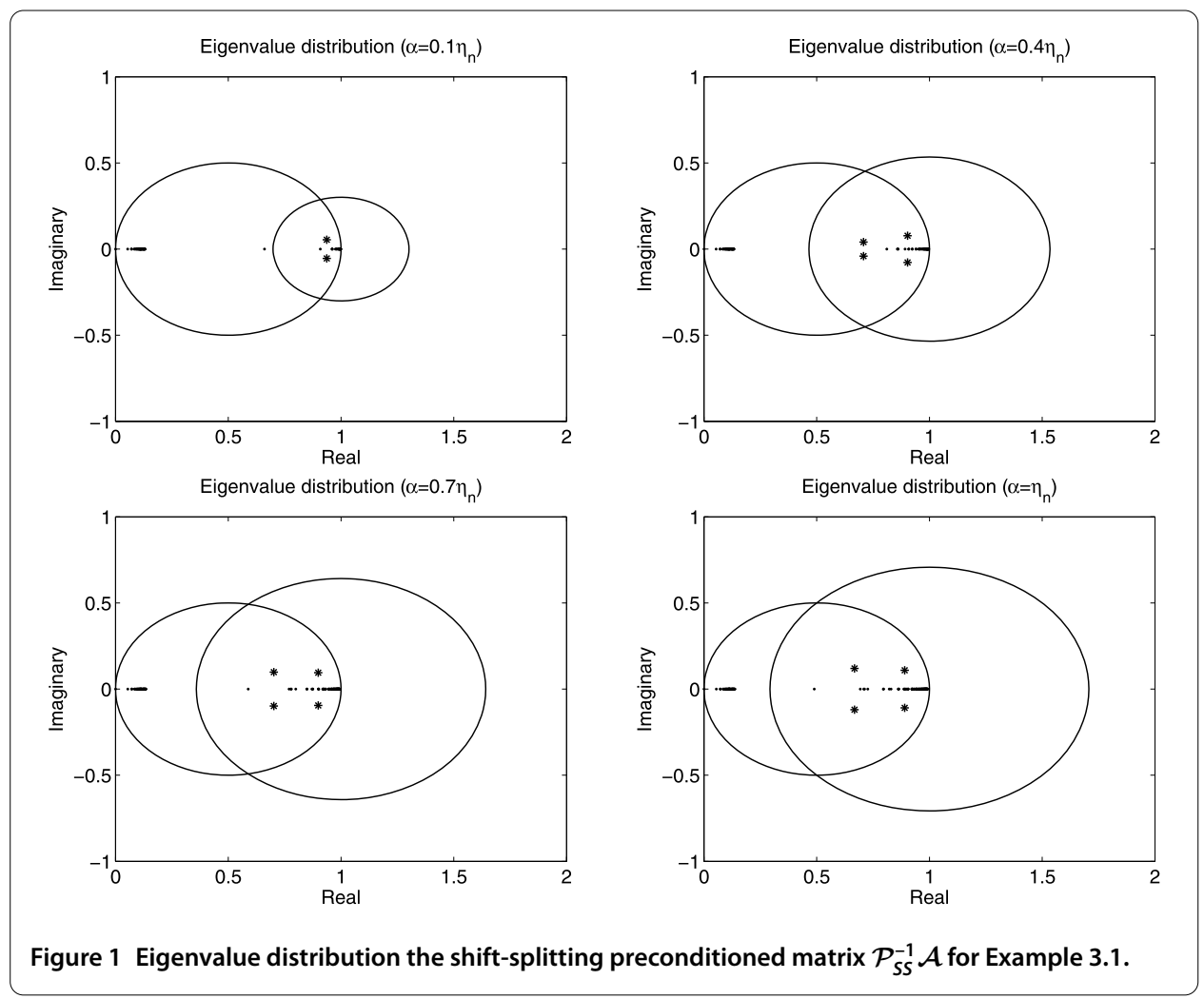

Table 2 Bounds of nonzero real eigenvalues of the preconditioned matrix $\mathcal{P}_{\text {SS }}^{-1} \mathcal{A}$ for Example 3.2

\begin{tabular}{lll}
\hline $\boldsymbol{( \alpha , \boldsymbol { \beta } )}$ & The estimated bound & The exact bound \\
\hline$(0.0077,0.0072)$ & {$[1.1108 \mathrm{e}-3,0.9995]$} & {$[0.1356,0.9990]$} \\
$(0.0307,0.0071)$ & {$[1.1108 \mathrm{e}-3,0.9981]$} & {$[0.1361,0.9960]$} \\
$(0.0538,0.0071)$ & {$[1.1108 \mathrm{e}-3,0.9966]$} & {$[0.1365,0.9930]$} \\
$(0.0768,0.0071)$ & {$[1.1108 \mathrm{e}-3,0.9952]$} & {$[0.1370,0.9900]$} \\
\hline
\end{tabular}

where $\Omega$ is a bounded domain, $v>0$ is the viscosity, the vector field $u$ stands for the velocity, and $p$ represents the pressure.

The IFISS software package developed by Elman et al. [22] is used to discretize the twodimensional Stokes equation (3.1) on the unit square domain. In actual computation, the Q2-Q1 mixed finite element method on uniform grid is used to generate discretizations. For simplicity, we set the viscosity values $v=1$ and take $16 \times 16$ grids to obtain the test matrix. Note that the rank of the matrix $B$ in the test nonsymmetric saddle-point matrix is $m-1$, which means that the discretized nonsymmetric saddle-point matrix $\mathcal{A}$ is singular. For the second example, the parameters $\alpha$ and $\beta$ are chosen by the same method as in Example 3.1. That is, we consider $\alpha=0.1 \eta_{n}, 0.4 \eta_{n}, 0.7 \eta_{n}, \eta_{n}$, and the parameters $\beta$ are obtained by the formula $\beta=\|B\|_{2}^{2} /\left\|\alpha I_{n}+A\right\|_{2}$. By computation we obtain that only one eigenvalue of the shift-splitting preconditioned matrix $\mathcal{P}_{S S}^{-1} \mathcal{A}$ is zero, which further confirms Theorem 2.1. In Table 2, we list the parameters $\alpha$ and $\beta$ for the shift-splitting preconditioner $\mathcal{P}_{S S}$, the estimated bounds, and the exact bounds of real eigenvalues of the shift-splitting preconditioned matrix $\mathcal{P}_{S S}^{-1} \mathcal{A}$ for Example 3.2. Figure 2 depicts the eigenval- 


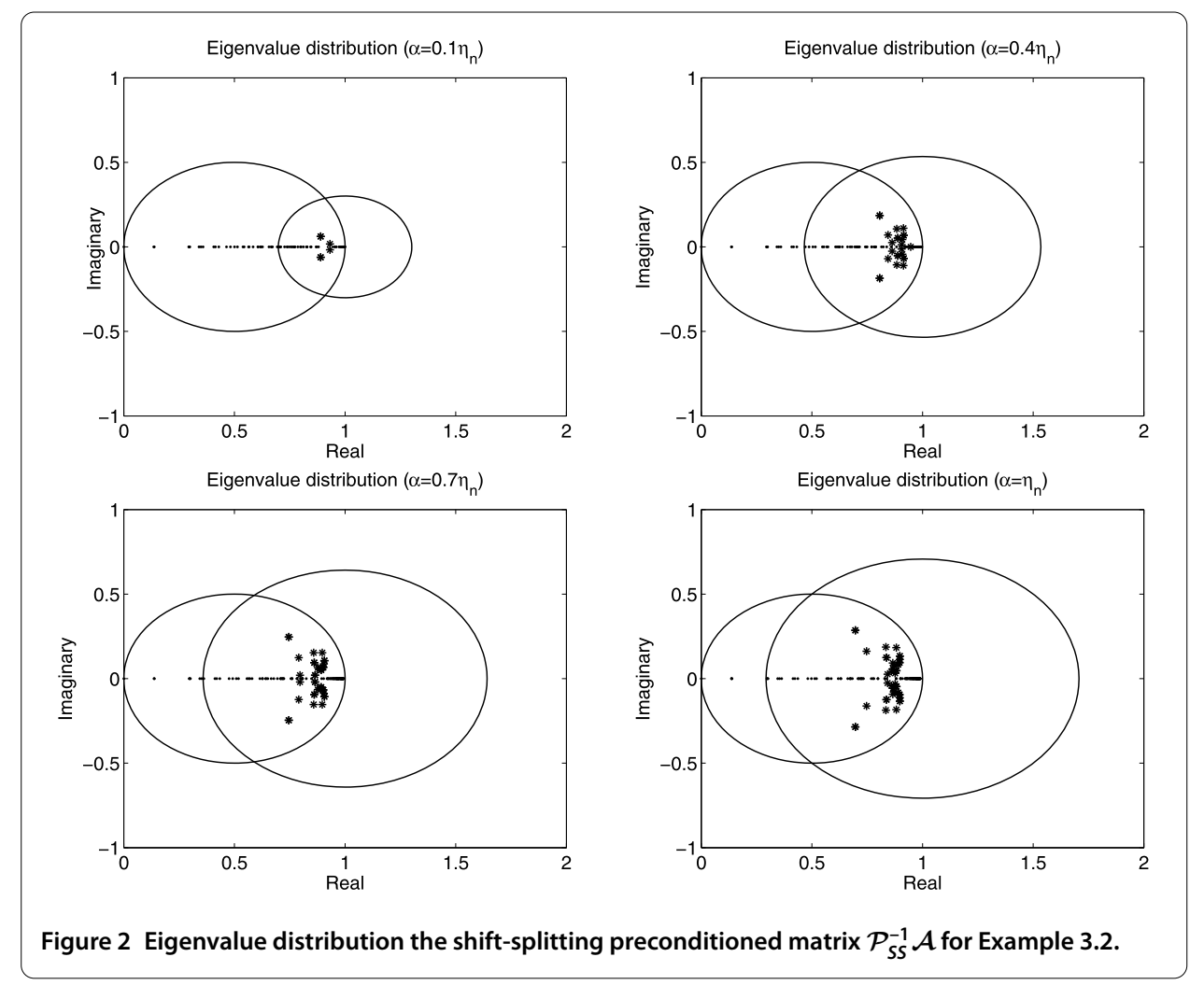

ues of the shift-splitting preconditioned matrix $\mathcal{P}_{S S}^{-1} \mathcal{A}$ together with the estimated circle. The numerical results presented in Table 2 and Figure 2 also coincide with our theoretical results in Theorem 2.3 and Theorem 2.4.

\section{Conclusion}

To further show the efficiency of the shift-splitting preconditioner for singular nonsymmetric saddle-point problems (1.1), the eigenvalue bounds of the shift-splitting preconditioned saddle-point matrix are studied in detail in this paper. Theoretical analysis shows that all eigenvalues having nonzero imaginary parts are located in an intersection of two circles and all nonzero real eigenvalues are located in a positive interval. Two numerical examples are presented to confirm the theoretical results. The numerical results show that the eigenvalue bounds are very sharp.

Competing interests

The authors declare that they have no competing interests.

Authors' contributions

The authors contributed equally to this work. All authors have read and approved the manuscript.

\section{Acknowledgements}

This work is supported by the National Natural Science Foundation of China (No. 11572210), the Natural Science Foundation of Jiangsu Province (No. BK20151272), the '333' Program Talents of Jiangsu Province (No. BRA2015356), and the Six Top Talents of Jiangsu Province (No. 2014-WLW-029).

Received: 18 July 2016 Accepted: 4 October 2016 Published online: 19 October 2016

\section{References}

1. Chen, C-R, Ma, C-F: A generalized shift-splitting preconditioner for singular saddle point problems. Appl. Math. Comput. 269, 947-955 (2015) 
2. Elman, HC, Silvester, DJ, Wathen, AJ: Finite Elements and Fast Iterative Solvers: With Applications in Incompressible Fluid Dynamics, 2nd edn. Oxford University Press, Oxford (2014)

3. Greif, C, Moulding, E, Orban, D: Bounds on eigenvalues of matrices arising from interior-point methods. SIAM J. Optim. 24, 49-83 (2014)

4. Cao, Y, Yao, L-Q, Jiang, M-Q, Niu, Q: A relaxed HSS preconditioner for saddle point problems from meshfree discretization. J. Comput. Math. 31, 398-421 (2013)

5. Benzi, M, Golub, GH, Liesen, J: Numerical solution of saddle point problems. Acta Numer. 14, 1-137 (2005)

6. Bai, Z-Z: Structured preconditioners for nonsingular matrices of block two-by-two structures. Math. Comput. 75, 791-815 (2006)

7. Bai, Z-Z: Eigenvalue estimates for saddle point matrices of Hermitian and indefinite leading blocks. J. Comput. Appl. Math. 237, 295-306 (2013)

8. Saad, Y: Iterative Methods for Sparse Linear Systems, 2nd edn. SIAM, Philadelphia (2003)

9. Pestana, J, Wathen, AJ: Natural preconditioning and iterative methods for saddle point problems. SIAM Rev. 57, 71-91 (2015)

10. Cao, Y, Du, J, Niu, Q: Shift-splitting preconditioners for saddle point problems. J. Comput. Appl. Math. 272, 239-250 (2014)

11. Cao, Y, Miao, S-X: On semi-convergence of the generalized shift-splitting iteration method for singular nonsymmetric saddle point problems. Comput. Math. Appl. 71, 1503-1511 (2016)

12. Shen, Q-Q, Shi, Q: Generalized shift-splitting preconditioners for nonsingular and singular generalized saddle point problems. Comput. Math. Appl. 72, 632-641 (2016)

13. Bai, Z-Z, Yin, J-F, Su, Y-F: A shift-splitting preconditioner for non-Hermitian positive definite matrices. J. Comput. Math. 24, 539-552 (2006)

14. Cao, Y, Tao, H-R, Jiang, M-Q: Generalized shift splitting preconditioners for saddle point problems. Math. Numer. Sin. 36, 16-26 (2014) (in Chinese)

15. Cao, Y, Li, S, Yao, L-Q: A class of generalized shift-splitting preconditioners for nonsymmetric saddle point problems. Appl. Math. Lett. 49, 20-27 (2015)

16. Olshanskii, MA, Simoncini, V: Acquired clustering properties and solution of certain saddle point systems. SIAM J. Matrix Anal. Appl. 31, 2754-2768 (2010)

17. Shen, $\mathrm{S}-\mathrm{Q}$, Huang, $\mathrm{T}-\mathrm{Z}, \mathrm{Yu}$, J: Eigenvalue estimates for preconditioned nonsymmetric saddle point matrices. SIAM J. Matrix Anal. Appl. 31, 2453-2476 (2010)

18. Notay, Y: A new analysis of block preconditioners for saddle point problems. SIAM J. Matrix Anal. Appl. 35, 143-173 (2014)

19. Cao, Y, Jiang, M-Q, Zheng, Y-L: A note on the positive stable block triangular preconditioner for generalized saddle point problems. Appl. Math. Comput. 218, 11075-11082 (2012)

20. Simoncini, V: Block triangular preconditioners for symmetric saddle-point problems. Appl. Numer. Math. 49, 63-80 (2004)

21. Axelsson, O: Unified analysis of preconditioning methods for saddle point matrices. Numer. Linear Algebra Appl. 22, 233-253 (2015)

22. Elman, HC, Ramage, A, Silvester, DJ: IFISS: a Matlab toolbox for modelling incompressible flow. ACM Trans. Math. Software 33, Article 14 (2007)

\section{Submit your manuscript to a SpringerOpen ${ }^{\odot}$ journal and benefit from:}

- Convenient online submission

- Rigorous peer review

- Immediate publication on acceptance

- Open access: articles freely available online

- High visibility within the field

- Retaining the copyright to your article

Submit your next manuscript at $>$ springeropen.com 